\title{
Vehicle Number Plate Detector
}

\author{
A. K. C. Varma \\ Associate Professor, ECE \\ Vishnu Institute of Technology
}

\author{
M. V. R. V. Prasad \\ Assistant Professor, ECE \\ Vishnu Institute of Technology
}

\author{
A. Madhavi \\ B.Tech, ECE \\ Vishnu Institute of Technology
}

\begin{abstract}
With an increase inthe number of vehicles on roads, it is getting difficult to manually enforce laws and traffic rules for smooth traffic flow. All these processes have a scope of improvement. In order to automate these processes and make them more effective, an algorithm is required to easily identify a vehicle. Therefore, we use number plate detection as vehicles in each country have a unique license number. An automated system can be achieved to detect the license plate of a vehicle and extract the characters from the license plate. The number plate can be used to retrieve more data about its owner, which can be used for further processing. Multiple methods are available to detect number plate regions and postprocessing methods are applied to merge all detected regions. In addition, trackers are used to limit the search region to certain areas in an image. This project suggests a different approach of detection using binarization and elimination of unnecessary regions from an image. The main purpose of this project is to recognize a license plate from an image provided by a camera. An efficient algorithm is developed to recognize a number plate in various luminance conditions. The system is achieved and simulated in Matlab and its performance is tested on real images.
\end{abstract}

\section{Keywords}

Automatedsystem,luminance conditions, Binarization,Number plate detection

\section{INTRODUCTION}

Number plate extraction is a difficult research area in the field of image processing. Many of automated systems have been matured but each has its pros and cons. It is assumed that this algorithm processed on images which have been captured from a fixed angle parallel to horizon in disparate brightness conditions. It is also assumed that the images of the static vehicles are captured from fixed distances.

An automated system which is developed using Matlab is used to capture the images and then those captured images are converted into grayscale images.After transformation, the dilation process is applied on an image and unnecessary holes in an image have been stuffed. After dilation, horizontal and vertical edge processing[1] are performed on images for finding boundaries and these images are processed using low pass filters. Low pass filters filter out unwanted region or unwanted noise from an image. After this filtering, the image is segmented and region of interest is extracted and the image is transformed into binary form. After binarization, each alphanumeric character on a license plate is extracted and then recognized[2] with template images of alphanumeric characters. After this, each alphanumeric character is gathered in a file and compared with the database to verify whether it is registered or not.

\section{LITERATURE SURVEY}

Most of the workspublishedon number plate detection originated in countries wherenumber plate design follows strict standards. There are abundantpaths, out ofthese, the basic path is the manual approach.

\section{Manual Approach:}

The license plate is accessed from the whole digital image of the vehicle in this method. The information can be obtained from this method in two levels. The first level is the binary mask stage which defines the region of the license plate and the second level is carrying out an AND operation. The AND operation is implemented between the binary mask and the binarized original vehicle image. This gives the localized license plate from a unified image.

\section{Intensity graph and Labeling approach:}

The data is extracted in this approach in two processes. They are: localization processand segmentation process.

\section{Localization process:}

In most of the vehicles, the plate is placed in the middle and lower part of the vehicle, although it may be contrasting in several cases. The height of the plate from the ground depends upon the vehicle design and in some cars the license plate placed in lower left or lower right part of the vehicle. Besides these abnormalities of the plate location, the distance between the camera and vehicle may also vary, and the localization of the plate inside the captured image plays a very important role. To improve the vehicle plate location accuracy, a new vehicle plate localization method[3] using properties of the captured image is proposed here. The horizontal and vertical plate localization is based on the properties of the image.

\section{Segmentation process:}

After the plate position has been estimated, inside the captured image, the part of the image corresponding to the plate section is represented by adopting only two intensity levels, zero(black) and one(white). The plate symbols are described using a library representation form. To extract the plate characters and also to identify those characters, a labeling process is applied to the binary image which is very useful technique to segment characters of different sizes with some inclinations because in this situation it is not possible to perform plate character segmentation[4] using only vertical and horizontal characters independently of the plate inclination, position, size and distance between the camera and plate position. Another method for segmenting the number plate is by using the principle of connectivity. In this stage, connected components are labeled first, for further analysis. In a digital black and white image, components can be 8 _connected or 4 _connected. After labeling components, each component will get a unique number which is very useful to segment each character. 


\section{METHODOLOGY}

The methodology is exposed in the flow chart. The flow chart is exposed in the figure 1 .

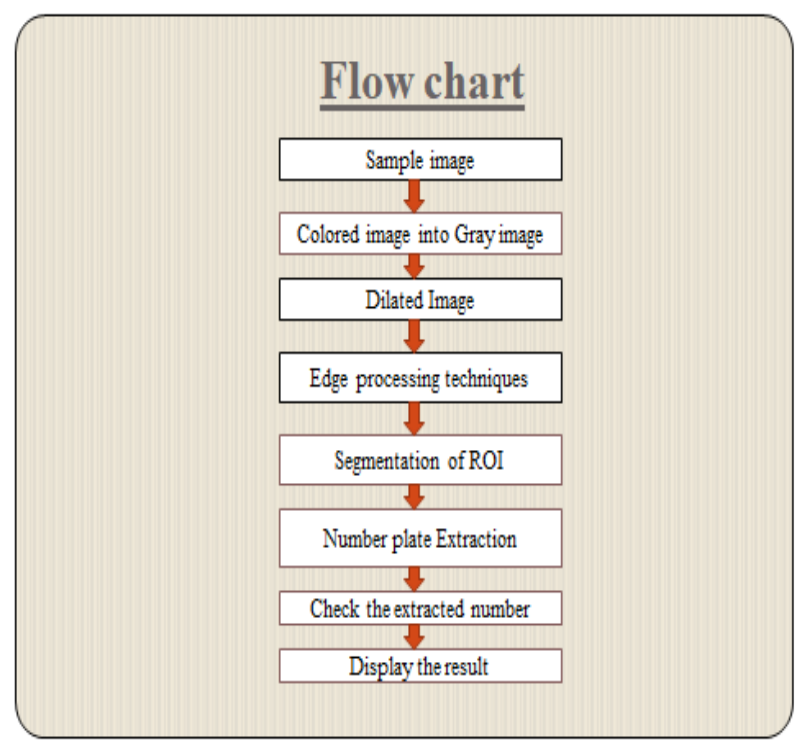

Fig 1. Flow chart

Image acquisition:

In this step, image is captured from a digital camera. An image should be taken from a fixed angle parallel to horizon. The vehicle should be stationary. The input image is shown in figure2.The image that is captured is absolutely unprocessed.

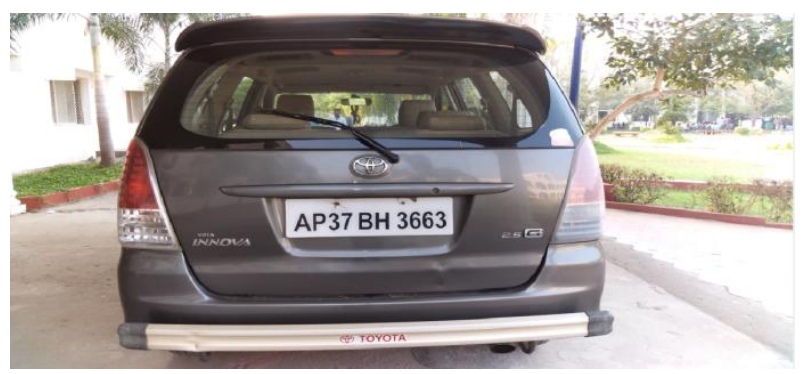

Fig 2. Sample image

Convert the input image into the gray image:

This algorithm works on Gray level image. Hence, the colored image is converted into the Grayscale image. The gray scale image is exposed in below figure 3 .

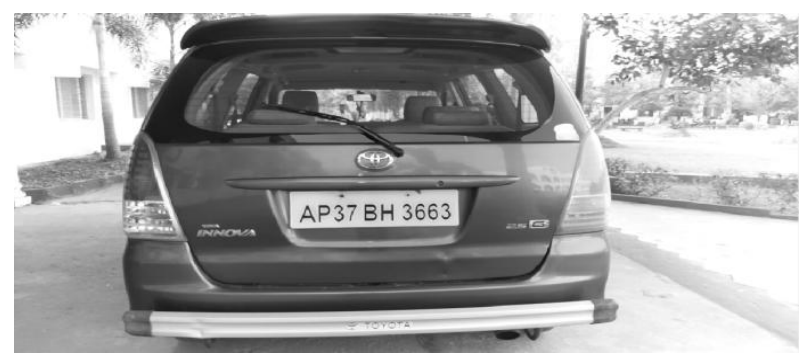

Fig 3. Color image to Grayscale image

\section{Dilated image:}

This process[5]fills the holes that are present in an image and thus improves the image quality and it also sharpens and increments the brightness of an image. In this step, every pixel is compared with neighboring pixels and its value is set equal to the utmost value of both adjoining pixels. The dilated image is exposed in figure 4.

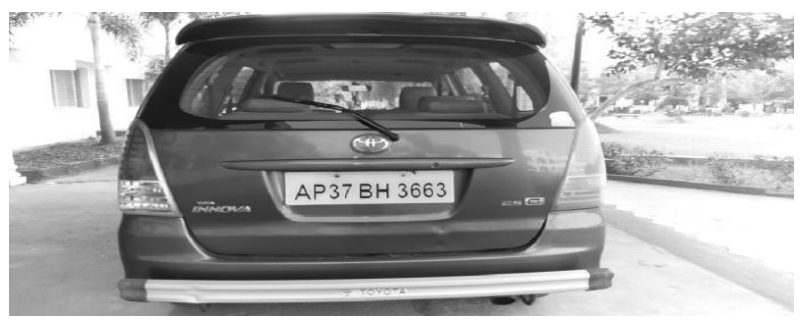

Fig 4. Dilated image

Edge processing histogram:

The horizontal and vertical histograms stand for row and column wise histograms. The below-mentioned histograms symbolize the row-wise and column-wise sum of differences of grayscale values among adjoining pixel values. Basically, the horizontal histogram is computed by passing it through every column and then the vertical histogram is computed by passing it through every row. In this step, the histogram values are averaged both sides and are processed using low pass filter to remove unwanted regions and then passed through bandpass filter to extract high probable regions. Passing histogram through low pass filter is exposed in figure $5 \& 6$.

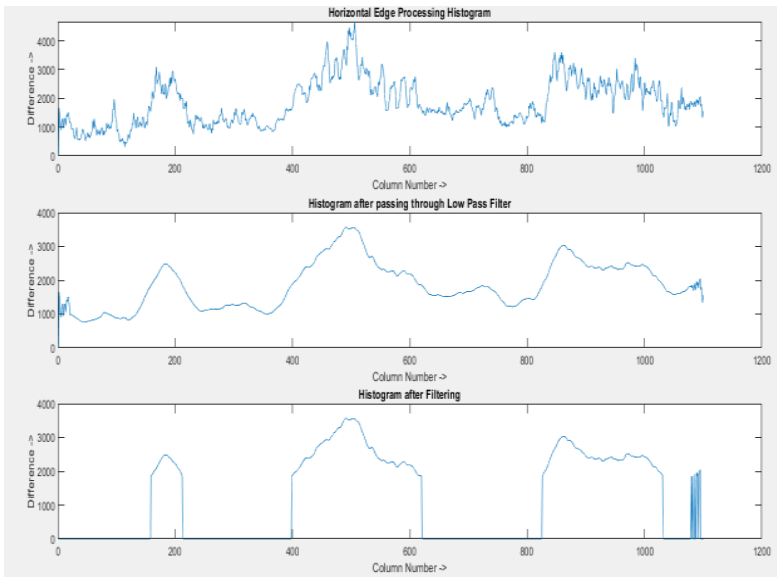

Fig 5. Horizontal edge processing

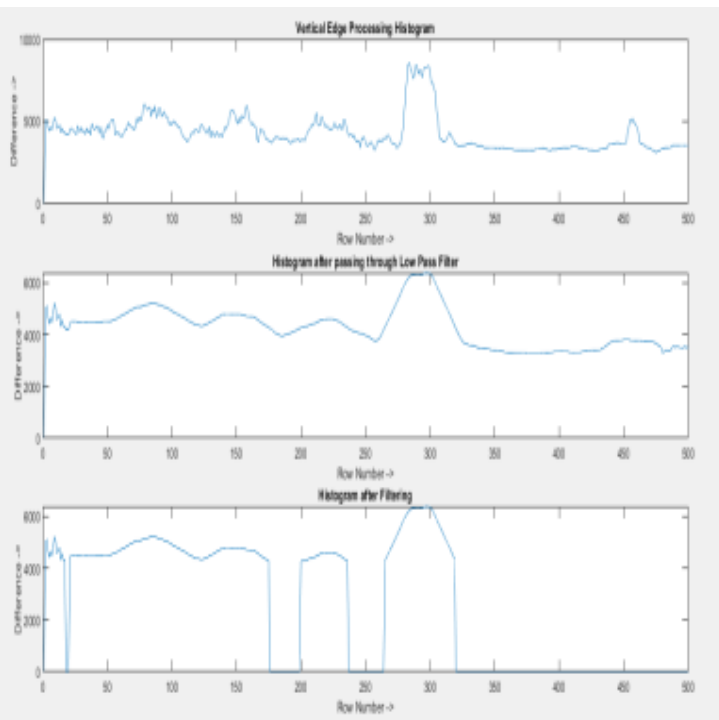

Fig 6. Vertical edge processing 


\section{Segmentation of ROI:}

Segmentation[4]finds a complete area that admits high chances of holding a license plate. In this step, coordinates of all such possible regions are stored in an array and also all undesirable areasare taken out from an image. The segmented regions are exposed in figure 7.

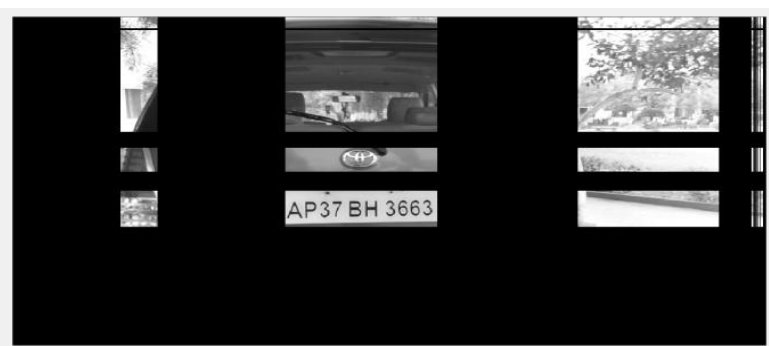

Fig 7. Segmented output

\section{Number plate extraction:}

The output of the segmentation process is all the regions that have the maximum probability of containing a license plate. Out of these regions, the one with the maximum histogram value is considered as the most probable candidate for the number plate. All the regions are processed row- wise and column-wise to find a common region having maximum horizontal and vertical histogram value. This is the region having highest probability of containing a license plate[6-7].

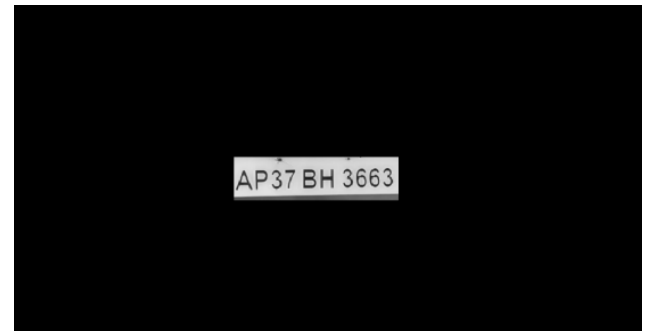

Fig 8. Extracted image

Check the extracted number:

The output of the segmented image accommodates entire region which is having an high chance of holding license plate and the region with apical histogram values are treated as utmost apparent contender plate region. The extracted number plate is exposed in figure 9 .

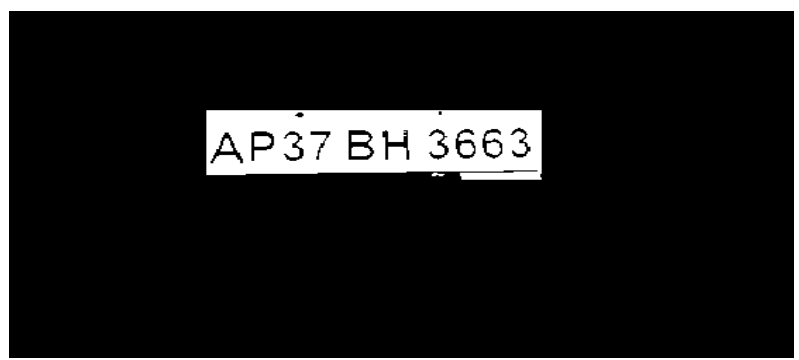

Fig 9. Binary Image of Extracted image

Bounding box image:

The bounding box[8] is used to measure the properties of the image region. Once a bounding box is created over each character and numbers presented on number plate, each character and number is separate out for recognition of number plate is shown in the figure 10. It displays the number plate of desired vehicle.

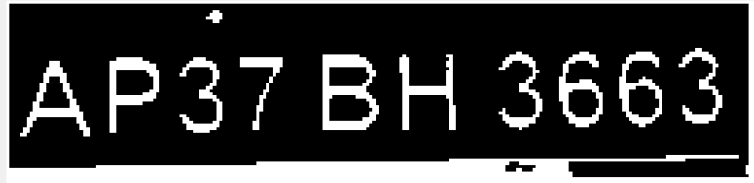

Fig 10. License plate with bounding box image

\section{Import data from ExcelSheet}

Initially, we store the number plates, owner names in excelsheet.After extracting the number plates from digital image, we store the extracted number plates. Now we compare the number plate result with our existing data by importing data from the excelsheet.Display the result as registered number with owner name if the result present in excel sheet.Display the result as not-registered number if the result does not exist in our excel sheet.

\section{GUI:}

GUIs (also known as graphical user interface) provides point and click control of software applications, eliminating the need to learn a language or type commands in order to run the application.GUIs are used because it makes things simple for the end-users of the program and if they were not used, people would have to work from the command line interface, which can be extremely difficult. The GUI based result is exposed in below figure 11

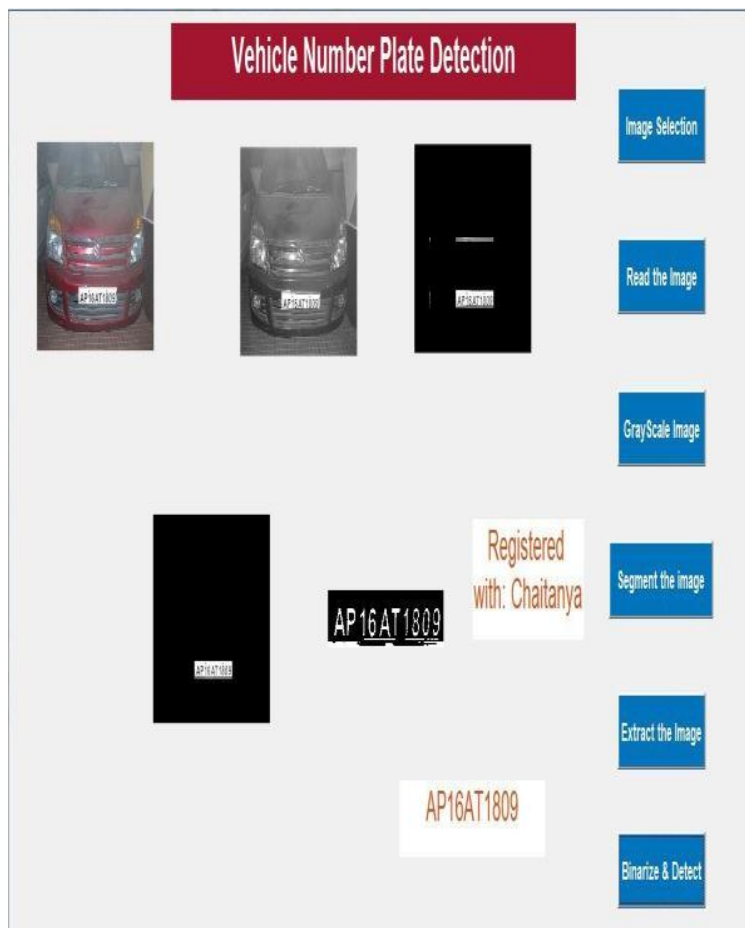

Fig. 11 GUI based result

\section{APPLICATIONS:}

Parking:

These are mainly used to reckon the lawn fee with the help of opening and closing times of the prepaid members.

\section{Access control:}

The gate naturally opens for the authorized representatives insecured areas thus replaces the security guard.

\section{Tolling:}


These are used near toll gates to count the travel fee with the help of car license number.

\section{CONCLUSION}

The paper title itself plays a key role in exposing the security alert. Number plate detection needs extremely high veracity when working on images of busy roads or parking areas. The system uses series of data for storage approaches for recognizing motor vehicles out of possession of database in $\mathrm{pc}$ and this entire scheme is implemented in MATLAB and its performance is tested on real-time pictures and also system robustness and speed can be increased if the high-resolution camera is used.

\section{REFERENCES}

[1] C. Chunyu, W. Fucheng, C. Baozhi and Z. Chen," Application of image processing to the vehicle license plate recognition," International Conference on Computer Science and Electronics Engineering, published by Allantis press, pp 2867-2869, 2013.

[2] Lekhana G.C, R.Srikantaswamy, "Real time license plate recognition system", International Journal of Advanced Technology \& Engineering Research (IJATER), National Conference on Emerging Trends in Technology (NCETTech) ISSN, Volume 2, Issue 4, ISSN No: 22503536, July 2012.
[3] Pandya and M Sing," Morphology based approach to recognize number plates in India," International Journal of Soft Computing and Engineering,Vol-1, Issue-3, pp 107-113, June2011.

[4] J.S. Chittode and R. Kate, "Number plate recognition using segmentation," International Journal of Engineering Research \& Technology, Vol. 1 Issue 9, November- 2012.

[5] H. Peng, F. Long and Z. Chi, "Document image recognition based on template matching of component block projections," IEEE transaction on Pattern Analysis and machine Intelligence, Vol. 25, no. 9, pp 1188-1192, sep 2003.

[6] Clemens Arth, Florian Limberger and Horst Bischof, "Real-Time License Plate Recognition on an Embedded DSP-Platform", Proceedings of IEEE conference on Computer Vision and Pattern Recognition, pp 1-8, June 2007.

[7] Pramod Kapadia, "Car License Plate Recognition Using Template Matching Algorithm", Master Project Report, California State University, Sacramento, Fall 2010.

[8] V. Lempitsky, P. Kohli, C. Rother, T. Sharp, "Image segmentation with a bounding box prior", MSR-TR2009-85. 